\title{
Modeling and Extraction of Parameters Based on Physical Effects in Bipolar Transistors
}

\author{
Agnes Nagy, Alicia Polanco, and Manuel Alvarez \\ Microelectronics Research Center, Antigua Carretera de Vento Km 8, Capdevila, P.O. Box 8016, Ciudad de la Habana 10800, Cuba \\ Correspondence should be addressed to Agnes Nagy, agnes.nagy@electrica.cujae.edu.cu \\ Received 30 September 2010; Revised 10 January 2011; Accepted 10 February 2011 \\ Academic Editor: Xiaobin Yuan \\ Copyright () 2011 Agnes Nagy et al. This is an open access article distributed under the Creative Commons Attribution License, \\ which permits unrestricted use, distribution, and reproduction in any medium, provided the original work is properly cited.
}

The rising complexity of electronic systems, the reduction of components size, and the increment of working frequencies demand every time more accurate and stable integrated circuits, which require more precise simulation programs during the design process. PSPICE, widely used to simulate the general behavior of integrated circuits, does not consider many of the physical effects that can be found in real devices. Compact models, HICUM and MEXTRAM, have been developed over recent decades, in order to eliminate this deficiency. This paper presents some of the physical aspects that have not been studied so far, such as the expression of base-emitter voltage, including the emitter emission coefficient effect $(n)$, physical explanation and simulation procedure, as well as a new extraction method for the diffusion potential $V_{\mathrm{DE}}(T)$, based on the forward biased base-emitter capacitance, showing excellent agreement between experimental and theoretical results.

\section{Introduction}

Every day, products and processes for microsystems become more complex and lead to high degrees of accuracy and stability. The simulation of the design must be very precise to increase the stability and reproducibility of the devices. Between the elements of electronic circuits used in microsystems, IC temperature sensors and temperature-compensated voltage references are frequently found. Reliable design of these circuits, fabricated in advanced bipolar and BiCMOS technologies has become seriously affected by the deficiencies of SPICE Gummel-Poon model (SGPM) as discussed by Schroter et al. [1] and need more physical-based and accurate models.

The base-emitter voltage, due to its extremely reproducible exponential characteristic, is the basic element in the design of I.C. temperature sensors and temperaturecompensated voltage references. To achieve the required accuracy and stability in these circuits, an accurate description of the base-emitter voltage temperature dependence $V_{\mathrm{BE}}(T)$ is needed.

The expressions of $V_{\mathrm{BE}}(T)$ reported in the literature have been obtained from collector current. The collector current of an NPN bipolar transistor working in the active region, at low current levels, neglecting emitter emission coefficient and direct and inverse Early effects, is given by Tsividis [2]:

$$
I_{C}(T)=I_{S}(T)\left[\exp \left(\frac{q V_{\mathrm{BE}}(T)}{k T}\right)-1\right],
$$

where $T$ is the absolute temperature, $V_{\mathrm{BE}}$ the base-emitter voltage, $q$ the electron charge, and $k$ the Boltzmann constant $\left(8.617 \times 10^{-5} \mathrm{eV} / \mathrm{K}\right)$. The -1 term can be neglected when the injected current is much greater than the saturation current $I_{S}(T)$.

The expression of $V_{\mathrm{BE}}(T)$ obtained from (1) by Tsividis [2] is known as the "accurate expression" and is given by

$$
\begin{aligned}
V_{\mathrm{BE}}(T)= & V_{G}(T)-V_{G}\left(T_{r}\right) \frac{T}{T_{r}} \\
& +V_{\mathrm{BE}}\left(T_{r}\right) \frac{T}{T_{r}}-(\eta-m) \frac{k T}{q} \ln \frac{T}{T_{r}},
\end{aligned}
$$

where $V_{\mathrm{BE}}\left(T_{\mathrm{r}}\right)$ and $V_{G}\left(T_{r}\right)$ are the base emitter and bandgap voltages at the reference temperature $T_{r}$. The accuracy of expression (2) depends on the accuracy of the bandgap voltage model $V_{G}(T)$ used. There are various models of $V_{G}(T)$ reported in the literature as discussed elsewhere [2-6], 


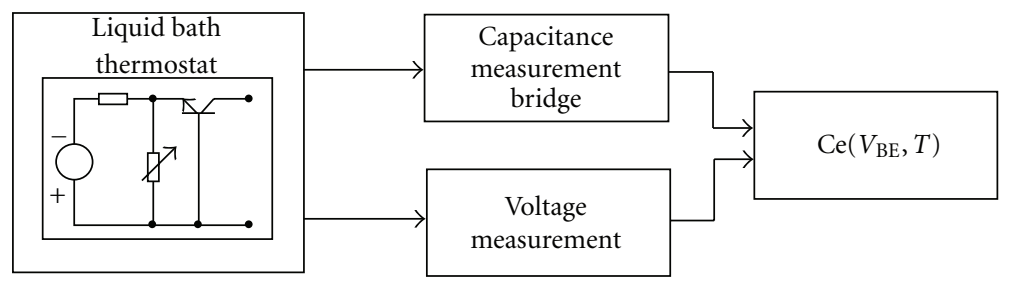

Figure 1: Measurement setup.

obtained by different methods for intrinsic silicon. In 1982, Meijer [7], using linear approximation for bandgap voltage $V_{G}(T)=V_{G 0}-\alpha T$ [5], obtained the following expression of $V_{\mathrm{BE}}(T)$ :

$$
V_{\mathrm{BE}}(T)=V_{G 0}\left[1-\frac{T}{T_{r}}\right]+\frac{T}{T_{r}} V_{\mathrm{BE}}\left(T_{r}\right)-(\eta-m) \frac{k T}{q} \ln \frac{T}{T_{r}},
$$

where $V_{G 0}$ is the extrapolated value of bandgap voltage at zero Kelvin and $\eta$ a parameter related to the dependence of global mobility with temperature through $\beta: \mu_{g} \propto T^{-\beta}$ as discussed by Slotboom and Graaff [8], which results from scattering mechanisms caused by thermal vibrations and ionized impurities as discussed by Amador et al. [9],

$$
\eta=4-\beta \text {. }
$$

The values of $V_{G 0}$ and $\eta$ can be obtained from experimental measurements of $V_{\mathrm{BE}}$ at three temperatures of the operating range. Experimental measurements of $\eta$ and $V_{G 0}$ in diffused wafers and in different types of bipolar transistors as reported elsewhere $[7,10]$ showed a negative correlation between both parameters as reported in the literature.

In order to improve $V_{\mathrm{BE}}(T)$ description, direct and inverse early effects have been discussed elsewhere $[11,12]$. Nevertheless, the emitter emission coefficient $n(T)$ and its influence on $V_{\mathrm{BE}}(T)$ has not been well studied up to now.

\section{Modeling and Extraction of Parameters}

The modeling and extraction of the parameters treated here are based on experimental measurements of forward biased base-emitter capacitance, using the measurement setup shown in Figure 1.

A Haake liquid bath thermostat guarantees temperature stability better than $0.05^{\circ} \mathrm{C}$. The DC measurements of $V_{\mathrm{BE}}$ were performed with a $61 / 2$ digits Agilent multimeter. Ce measurement error in the bridge due to parallel base-emitter diode conductance is negligible except near the maximum value of $\mathrm{Ce}$, where it is only $2 \%$. Therefore, no corrections are introduced in the experimental values of $\mathrm{Ce}$.

From experimental measurements of Ce realized with MAT01 standard bipolar transistors, an unexpected exponential increase under significant forward bias has been observed. This behavior is not considered by Ideal, GummelPoon nor the first MEXTRAM and HICUM Ce models as can be seen elsewhere [13-15]. It may be seen from Figure 2 that the three models fail to follow the exponential behavior

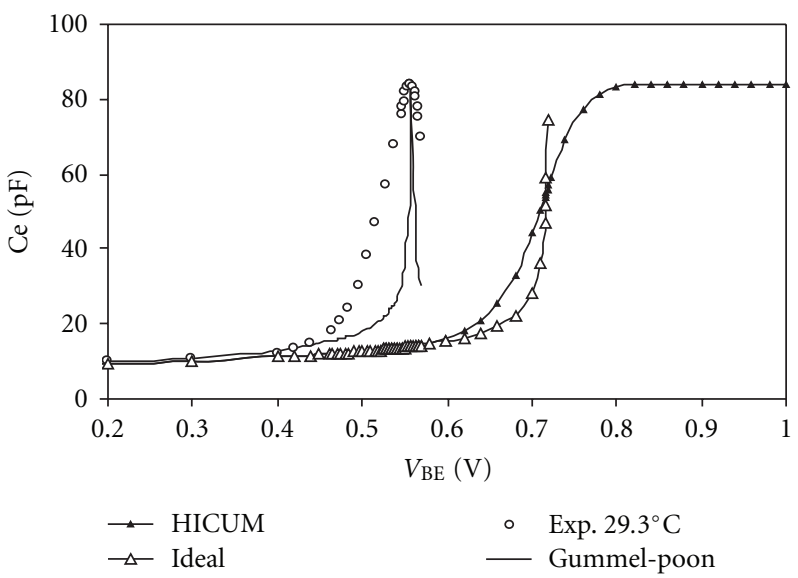

Figure 2: Experimental and simulated values of Ce at $29.3^{\circ} \mathrm{C}$.

of $\mathrm{Ce}$ and begin to increase at higher bias voltages. For this reason, it was necessary to develop a new forward biased emitter capacitance model of bipolar junction transistors, published by Nagy et al. [16], which includes a region with exponential behavior of the emitter capacitance Ce as forward bias increases. The physical explanation of the exponential increase of $\mathrm{Ce}$ was found in the theory for the transition region of the $\mathrm{p}-\mathrm{n}$ junction as discussed by Shockley et al. [17]. Modern compact bipolar transistor models such as MEXTRAM and HICUM as discussed elsewhere [18, 19] take the neutral charge into account using a charge-transit time model.

Modeling and extraction of parameters treated here are based on the new forward bias base-emitter capacitance model as discussed by Nagy et al. [16]. A brief description is given as follows.

\subsection{Brief Description of New Bipolar Transistor Base-Emitter} Capacitance Model. The new model is built using two expressions linked with a smoothing function: the threeparameter Ideal expression that predominates at low forward bias and the neutral capacitance expression that predominates as forward bias increases, as discussed by Nagy et al. [16]. The fit of the new Ce model to experimental results is notable. This model of Ce in function of base-emitter voltage and temperature is given by

$$
\mathrm{Ce}\left(V_{\mathrm{BE}}, T\right)=f\left(V_{\mathrm{BE}}\right) C_{\mathrm{Sc}}(T)+C_{n}(T),
$$


where $f\left(V_{\mathrm{BE}}\right)$ is a smoothing function defined for a voltage interval $\left[V_{\mathrm{BE} 1}, V_{\mathrm{BE} 2}\right]$ given by

$$
f\left(V_{\mathrm{BE}}\right)=\frac{1}{1+e^{\lambda\left(V_{\mathrm{BE}}-\alpha\right)}},
$$

where

$$
\alpha=\frac{V_{\mathrm{BE} 1}+V_{\mathrm{BE} 2}}{2}, \quad \lambda=\frac{\ln 99}{\delta}, \quad \delta=\frac{V_{\mathrm{BE} 2}-V_{\mathrm{BE} 1}}{2},
$$

(i) $V_{\mathrm{BE} 2}$ is the base-emitter voltage $V_{\mathrm{BE} \max }$ at maximum $\mathrm{Ce}$,

(ii) $V_{\mathrm{BE} 1}$ is the top value of base-emitter voltages that satisfy the space charge condition taking into account experimental measurements.

In the Ideal or space charge capacitance model,

$$
C_{\mathrm{SC}}(T)=\frac{C_{j e 0}(T)}{\left[1-V_{\mathrm{BE}} / V_{\mathrm{DE}}(T)\right]^{Z}},
$$

where $C_{j e 0}(T)$ is the zero-bias capacitance.

The temperature dependence of the zero-bias emitter capacitance is given by

$$
C_{j e 0}(T)=C_{j e 0}\left(T_{r}\right)\left[\frac{V_{\mathrm{DE}}\left(T_{r}\right)}{V_{\mathrm{DE}}(T)}\right]^{Z} .
$$

It is also known as reported by Schroter et al. [13] that the diffusion voltage $V_{\mathrm{DE}}$ depends on temperature through

$$
V_{\mathrm{DE}}(T)=V_{G 0}\left[1-\frac{T}{T_{r}}\right]+V_{\mathrm{DE}}\left(T_{r}\right) \frac{T}{T_{r}}-3 V_{T} \ln \frac{T}{T_{r}} .
$$

The expression of the neutral capacitance was obtained using Shockley's theory on $\mathrm{p}-\mathrm{n}$ junction transition region capacitance. For the case of a bipolar transistor, it can be written as shown by Nagy et al. [16] as

$$
C_{n}\left(V_{\mathrm{BE}}, T\right)=C_{S}(T)\left[\frac{V_{\mathrm{DE}}(T)-V_{\mathrm{BE}}}{k T / q}-1\right] \exp \left(\frac{q V_{\mathrm{BE}}}{k T}\right)
$$

with

$$
C_{S}(T)=C_{S}\left(T_{r}\right)\left(\frac{T}{T_{r}}\right)^{2} \exp \left[\frac{q V_{G 0}}{k}\left(\frac{1}{T_{r}}-\frac{1}{T}\right)\right],
$$

where $T_{r}$ is a reference temperature and $V_{G 0}$ is the zero Kelvin extrapolated bandgap voltage.

Based on expression (11) and the condition for a maximum capacitance $\mathrm{d} C_{n} / \mathrm{d} V_{\mathrm{BE}}=0$, an important relationship between $V_{\mathrm{BE} \max }$ and $V_{\mathrm{DE}}$ (that allows the experimental determination of $V_{\mathrm{DE}}$ at any temperature) is found,

$$
V_{\mathrm{BE} \max }(T)=V_{\mathrm{DE}}(T)-\frac{2 k T}{q} .
$$

The simulation of experimental measured Ce values at $39.3^{\circ} \mathrm{C}$ was used to assess the temperature dependence of

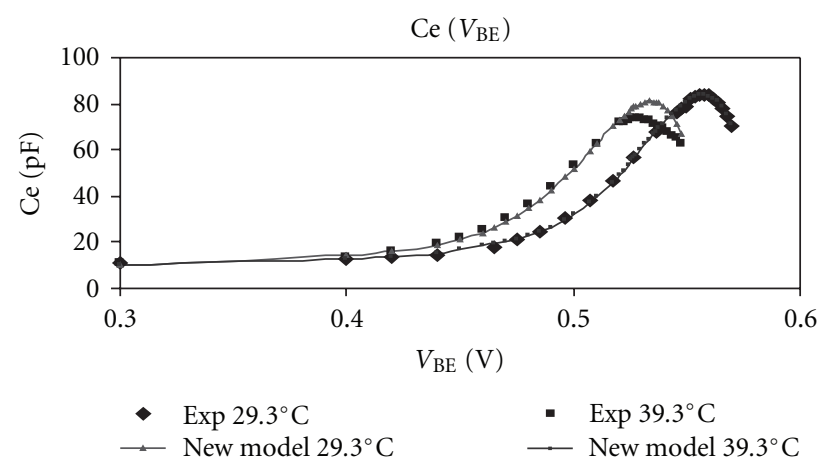

Figure 3: Experimental and simulated values of Ce at $29.3^{\circ} \mathrm{C}$ and $39.3^{\circ} \mathrm{C}$ with the new model.

the new Ce model. The measured and simulated values of Ce with the new model at two temperatures are shown in Figure 3.

The simulation parameters, extracted from the experimental measurements at $29.3^{\circ} \mathrm{C}$, are $C_{j e 0}\left(T_{r}\right)=8.52 \mathrm{pF}$, $z=0.329, V_{\mathrm{DE}}\left(T_{r}\right)=0.610 \mathrm{~V}, \mathrm{Ce}_{\max }\left(T_{r}\right)=84 \mathrm{pF}, C_{s}\left(T_{r}\right)=$ $4.259 \times 10^{-8} \mathrm{pF}$, and $V_{\mathrm{BE} \max }\left(T_{r}\right)=0.558 \mathrm{~V}$. The limits of the smoothing function interval at $T=T_{r}=29.3^{\circ} \mathrm{C}$ are $V_{\mathrm{BE} 1}=0.400 \mathrm{~V}$ and $V_{\mathrm{BE} 2}=0.558 \mathrm{~V}$ while at $T=39.3^{\circ} \mathrm{C}$ are $V_{\mathrm{BE} 1}=0.372 \mathrm{~V}$ and $V_{\mathrm{BE} 2}=0.535 \mathrm{~V}$. The measured and predicted values using the new model are summarized in Table 1.

A very good $1 \%$ extrapolation accuracy is observed except for $\mathrm{Ce}_{\max }$ with almost $8 \%$ error that can be attributed to $\pm 5 \%$ time and temperature drift of the capacitance bridge during measurements.

\section{Effect of Emitter Emission Coefficient}

The variation of emitter emission coefficient $(n)$ with temperature has been measured and reported elsewhere [20, $21]$. At very low temperature $(230 \mathrm{~K}-300 \mathrm{~K})$, an increase of the value of $n$ was found. Until now, there is no satisfactory explanation for this phenomenon.

In this paper, a physical approach of $n(T)$ behavior through of emitter-base capacitance $\mathrm{Ce}\left(V_{\mathrm{BE}}, T\right)$ is presented. Considering the effect of emission coefficient $n(T)$, the collector current $I_{C}(T)$ can be written as

$$
I_{C}(T)=I_{S}(T)\left[\exp \left(\frac{q V_{\mathrm{BE}}(T)}{n(T) k T}\right)-1\right],
$$

from which $V_{\mathrm{BE}}(T)$ expression can be obtained as

$$
\begin{aligned}
V_{\mathrm{BE}}(T)= & n(T)\left[V_{G}(T)-V_{G}\left(T_{r}\right) \frac{T}{T_{r}}\right] \\
& +V_{\mathrm{BE}}\left(T_{r}\right) \frac{T}{T_{r}} \frac{n(T)}{n\left(T_{r}\right)}-n(T)(\eta-m) \frac{k T}{q} \ln \frac{T}{T_{r}} .
\end{aligned}
$$

Equation (15) shows that $V_{\mathrm{BE}}(T)$ is affected by both the value of emission coefficient and its variation with temperature. 
TABLE 1: Values measured and predicted with the new model.

\begin{tabular}{lcc}
\hline Measured values at $29.3^{\circ} \mathrm{C}$ & Predicted values at $39.3^{\circ} \mathrm{C}$ & Measured values at $39.3^{\circ} \mathrm{C}$ \\
\hline$V_{\mathrm{BE} \max }=0.558 \mathrm{~V}$ & $V_{\mathrm{BE} \max }=0.5346 \mathrm{~V}$ & $V_{\mathrm{BE} \max }=0.532 \mathrm{~V}$ \\
$\mathrm{Ce}_{\max }=84 \mathrm{pF}$ & $\mathrm{Ce}_{\max }=81.54 \mathrm{pF}$ & $\mathrm{Ce}_{\max }=75.2 \mathrm{pF}$ \\
$V_{\mathrm{DE}}=0.610 \mathrm{~V}$ & $V_{\mathrm{DE}}=0.5885 \mathrm{~V}$ & $V_{\mathrm{DE}}=0.586 \mathrm{~V}$ \\
$C_{S}=4.259 \times 10^{-8} \mathrm{pF}$ & $C_{S}=19.47 \times 10^{-8} \mathrm{pF}$ & $C_{S}=19.7 \times 10^{-8} \mathrm{pF}$ \\
\hline
\end{tabular}

Three cases can be differentiated:

(a) $n=1$ for all of the temperature range: in this case, (15) is reduced to the expression reported in the literature;

(b) $n>1$ and constant for all of the temperature range: in this case $n(T)=n\left(T_{r}\right)$, so only the linear term is not affected;

(c) $n>1$ and varies with $T$ : in this case, all of the terms are affected.

This makes necessary to characterize the variation of $n$ as a function of bias and temperature.

3.1. Physical Approach to Emitter Emission Coefficient. Many authors consider the emission coefficient, as in the SPICE Gummel-Poon model (SGP), a constant value close to the unity to explain the nonideality of the exponential dependence of collector current $I_{C}$ on emitter-base voltage as discussed elsewhere [2, 10] (see (14)). Nevertheless, as was discussed by Gummel and Poon [14], in the original Gummel-Poon model $n$ is not a parameter, and it is defined at given $V_{\mathrm{CB}}$ and $T$ as the reciprocal of the slope of the Gummel plot given at low level injection by

$$
n(T)=1+\frac{k T \mathrm{Ce}}{q Q_{b 0}} .
$$

Extending the Gummel-Poon model as discussed by Nagy et al. [22] definition of $n$ in temperature results in

$$
n(T)=1+\frac{k T \operatorname{Ce}(T)}{q Q_{b 0}},
$$

where $\operatorname{Ce}(T)$ is the emitter capacitance and $Q_{b 0}$ the zerobias charge in the base, which can be considered constant. This expression allows a physical approach of its behavior with forward bias and temperature through $\mathrm{Ce}(T)$, which has been widely studied elsewhere $[13,14,23,24]$.

3.2. Simulation of Emitter Emission Coefficient. A new simulation procedure was elaborated to characterize the behavior of the emission coefficient of a bipolar transistor (biased at constant collector current) with the temperature. This procedure uses the original Gummel-Poon's expressions of $n$ including its temperature dependence (see (17)) and the new Ce model developed as a function of base-emitter voltage and temperature.

A flow chart showing the simulation procedure used to obtain $n(T)$ is presented in Figure 4, which includes experimental and simulated values of $\mathrm{Ce}$ at $29.3^{\circ} \mathrm{C}$.
3.3. Simulation Results. The emission coefficient was calculated in the temperature range of $240 \mathrm{~K}-300 \mathrm{~K}$ using (17), where the value of $Q_{b 0}$ was considered constant and equal to $6.0 \mathrm{pF}$. The simulation parameters extracted from experimental Ce measurements at reference temperature $\left(29.3^{\circ} \mathrm{C}\right)$ were the following: $C_{j e 0}\left(T_{r}\right)=8.52 \mathrm{pF}, V_{\text {DEideal }}\left(T_{r}\right)=$ $0.719 \mathrm{~V}, z=0.329, C_{S}\left(T_{r}\right)=4.259 \times 10^{-8} \mathrm{pF}$, and $V_{\text {DE neutral }}\left(T_{r}\right)=0.61, \mathrm{Ce}_{\max }\left(T_{r}\right)=84 \mathrm{pF}$, and $V_{\mathrm{BE} \max }\left(T_{r}\right)=$ $0.558 \mathrm{~V}$. Bias conditions for simulation at $T_{r}$ were given by $V_{\mathrm{BE}}=0.5 \mathrm{~V}$ and $V_{\mathrm{BE}}=0.46 \mathrm{~V}$, both selected into the region with exponential behavior of the emitter capacitance Ce as forward bias increases.

Simulation results of MAT01 standard bipolar transistor with $\eta=3.54, V_{G 0}=1.185 \mathrm{~V}$ and vertical PNP transistors fabricated in $0.5 \mu \mathrm{m}$ CMOS technology with $\eta=5.1, V_{G 0}=$ $1.12046 \mathrm{~V}$ at two bias levels are shown in Figures 5 and 6.

The temperature sensitivity of $n$ is related to $\mathrm{TCe}(T)$ product, as can be seen in (17). It implies a dependence of emitter area confirmed through the experimental measurements of $n(T)$ at low temperatures for different emitter areas as discussed by Wang and Meijer [20]. From the simulation results presented, it can be observed that the increase of $n$ at low temperature also depends on bias conditions and technological parameters $\eta$ and $V_{G 0}$. In the case of vertical pnp transistors fabricated with $0.5 \mu \mathrm{m}$ CMOS technology, the simulated behaviour of $n(T)$ shows a similar trend than measured data reported.

3.4. Assessment of $n(T)$ Effect on $V_{B E}(T)$. The estimation of emitter emission coefficient effect was obtained using the linear approximation for $V_{G}(T)$ in (15) of $V_{\mathrm{BE}}(T)$, resulting in

$$
\begin{aligned}
V_{\mathrm{BE}}(T)= & n(T) V_{G 0}\left[1-\frac{T}{T_{r}}\right] \\
& +V_{\mathrm{BE}}\left(T_{r}\right) \frac{T}{T_{r}}\left[\frac{n(T)}{n\left(T_{r}\right)}\right]-n(T) \eta \frac{k T}{q} \ln \frac{T}{T_{r}},
\end{aligned}
$$

where $n(T) V_{G 0}=V_{G 0}^{\prime}$ and $n(T) \eta=\eta^{\prime}$ represent the best fitting parameters for $V_{\mathrm{BE}}(T)$, extracted from experimental measurements. Now (18) is modified as

$$
\begin{aligned}
V_{\mathrm{BE}}(T)= & V_{G 0}^{\prime}\left[1-\frac{T}{T_{r}}\right] \\
& +V_{\mathrm{BE}}\left(T_{r}\right) \frac{T}{T_{r}}\left[\frac{n(T)}{n\left(T_{r}\right)}\right]-\eta^{\prime} \frac{k T}{q} \ln \frac{T}{T_{r}} .
\end{aligned}
$$




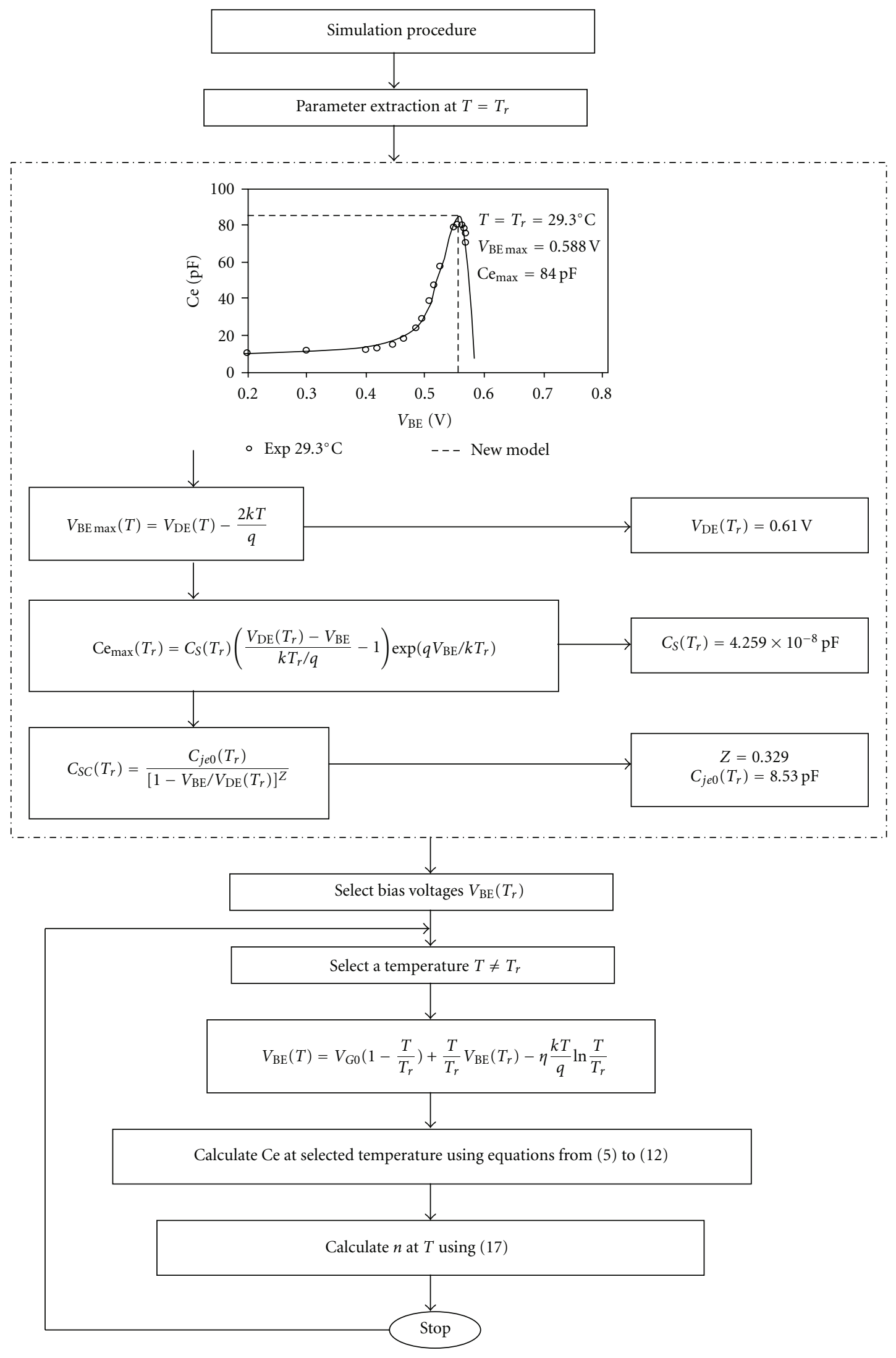

FIgURE 4: Flow chart: simulation procedure to obtain $n(T)$. 
TABLE 2: Calculated values of $\Delta V_{\mathrm{BE}}$ and its equivalent temperature error.

\begin{tabular}{|c|c|c|c|c|c|c|c|}
\hline \multicolumn{6}{|c|}{ Data at $T_{r}=300 \mathrm{~K}$ and $T=240 \mathrm{~K}$} & \multicolumn{2}{|c|}{ Results } \\
\hline Technology & $V_{\mathrm{BE}}\left(T_{r}\right) V$ & $n\left(T_{r}\right)$ & $n(T)$ & $V_{G 0}^{\prime} \mathrm{V}$ & $\eta^{\prime}$ & $\Delta V_{\mathrm{BE}}(T) \mathrm{mV}$ & $\Delta \mathrm{Teq}^{\circ} \mathrm{C}$ \\
\hline \multirow{2}{*}{ NPN (Bipolar) } & 0.46 & 1.07496 & 1.07764 & 1.12046 & 5.1 & 1.711 & 0.855 \\
\hline & 0.50 & 1.12459 & 1.15790 & 1.12046 & 5.1 & 10.40 & 5.2 \\
\hline \multirow{2}{*}{ PNP (CMOS) } & 0.46 & 1.07472 & 1.07003 & 1.18500 & 3.54 & -1.606 & 0.803 \\
\hline & 0.50 & 1.12344 & 1.12837 & 1.18500 & 3.54 & 1.756 & 0.878 \\
\hline
\end{tabular}

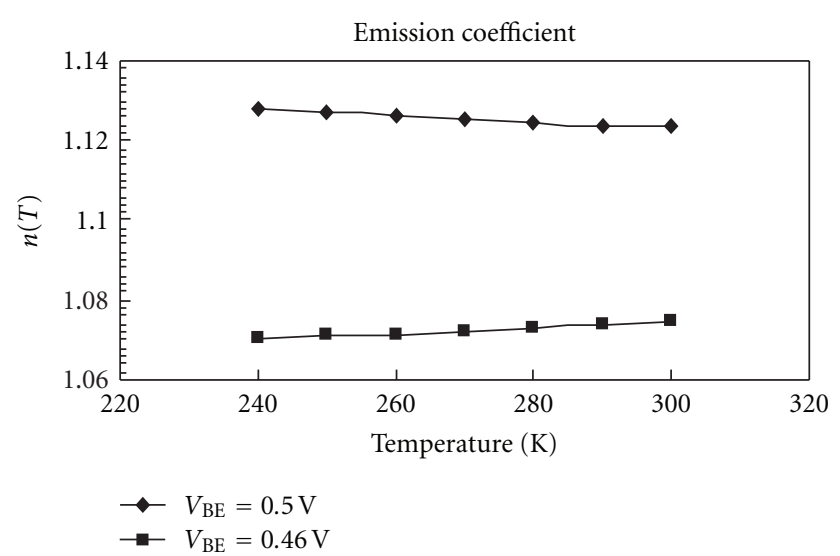

FIGURE 5: Variation of $n(T)$ of standard bipolar MAT01 transistor with $\eta=3.54, V_{G 0}=1.185 \mathrm{~V}$.

The error $\Delta V_{\mathrm{BE}}(T)$ introduced, neglecting the variation of emission coefficient with temperature, was estimated for transistors fabricated with different technologies, using (19). The results are shown in Table 2 , where the error $\Delta V_{\mathrm{BE}}(T)$, expressed in equivalent temperature, was estimated considering a typical variation of $-2 \mathrm{mV} /{ }^{\circ} \mathrm{C}$ for base-emitter voltage.

These results assess the convenience of taking into account the variation of emission coefficient with temperature in the design of circuits based on base-emitter voltage, as I.C. temperature sensors and compensated bandgap voltage references.

\section{Built-in Voltage}

The difference of potential that exists across a pn junction in equilibrium, known as contact potential, diffusion potential, or "built-in potential," $V_{\mathrm{DE}}$, is a fundamental parameter in the theoretical study and modeling of the pn junction and semiconductor devices. This potential is present in the theoretical expressions of space charge, in Ideal model of space charge capacitance of the pn junction in the emitter-base capacitance Poon-Gummel model, HICUM and Mextram compact models as discussed elsewhere [15, 23-26]. The determination of $V_{\mathrm{DE}}$ 's value, being a parameter associated with the equilibrium, is only possible indirectly, from the measurement of the capacity of the corresponding junction.

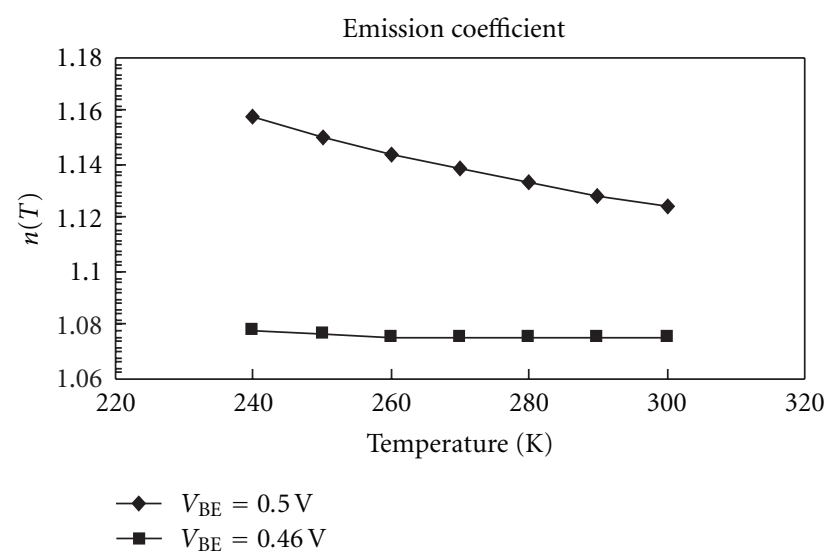

FIGURE 6: Variation of $n(T)$ for vertical PNP transistors in $0.5 \mu \mathrm{m}$ CMOS technology with $\eta=5.1, V_{G 0}=1.12046 \mathrm{~V}$.

As was reported by Schroter [13], the diffusion voltage $V_{\mathrm{DE}}$ depends on temperature through

$$
V_{\mathrm{DE}}(T)=V_{G 0}\left[1-\frac{T}{T_{r}}\right]+V_{\mathrm{DE}}\left(T_{r}\right) \frac{T}{T_{r}}-3 V_{T} \ln \frac{T}{T_{r}}
$$

where $V_{\mathrm{DE}}\left(T_{r}\right)$ is the diffusion potential at reference temperature $T_{r}$.

4.1. Measurement of $V_{D E}$. The determination of $V_{\mathrm{DE}}(T)$ values is based on (13) using experimental measured values of $V_{\mathrm{BE} \max }(T)$, obtained with the measurement setup in Figure 1.

For the comparison of the experimental and theoretical behavior of the diffusion potential with temperature, the theoretical values of $V_{\mathrm{DE}}(T)$ by means of expression (20) were calculated, taking $V_{\mathrm{DE}}\left(T_{r}\right)$ as the experimental value of $V_{\mathrm{DE}}(T)$ at the lowest temperature of measurement and the value of $V_{G 0}=1.185 \mathrm{~V}$ brought by Meijer [27] for the transistor MAT01. The results are plotted in Figure 7.

Results show an excellent agreement between theory and experiments with a maximum error lower than $2 \%$. These results validate the method of measurement used for the determination of diffusion potential and assess (20) to describe the behavior of the diffusion potential with temperature. 


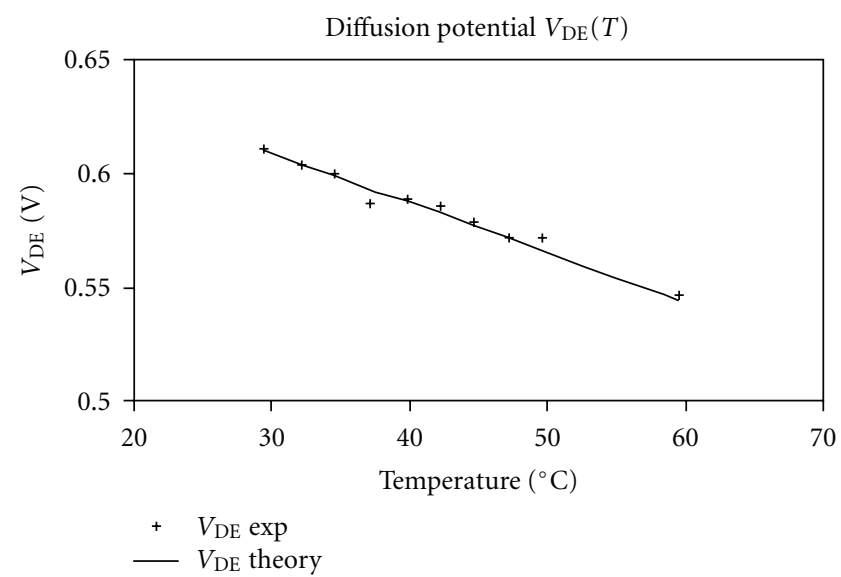

FIGURE 7: Variation of diffusion potential versus temperature.

\section{Conclusions}

A physical point of view in the analysis of the approximations used in $V_{\mathrm{BE}}(T)$ expression allows to reach an improved description of base-emitter voltage temperature dependence, considering the effect of emitter emission coefficient $n$ and its direct dependence on base-emitter capacitance Ce.

The obtained results allow a physical approach to $n(T)$ behavior with forward bias and temperature through $\mathrm{Ce}(T)$ and a procedure to simulate $n(T)$ values, using a new Ce model.

The estimation of error $\Delta V_{\mathrm{BE}}(T)$ introduced neglecting the emission coefficient shows that it is convenient to take into account the variation of emission coefficient with temperature in the design of circuits based on baseemitter voltage, as I.C. temperature sensors and compensated bandgap voltage references.

A new method for measurement of diffusion potential $V_{\mathrm{DE}}(T)$ based on the theory of pn junction neutral capacitance and the measurement of emitter capacitance Ce versus $V_{\mathrm{BE}}$, was developed. The measurements of $V_{\mathrm{DE}}(T)$ show good concordance with theoretical behavior, confirming the validity of both, the method used and the theoretical equation reported in the literature.

\section{References}

[1] M. Schroter, S. Lehmann, S. Frégonèse, and T. Zimmer, "A computationally efficient physics-based compact bipolar transistor model for circuit design-part I: model formulation," IEEE Transactions on Electron Devices, vol. 53, no. 2, pp. 279286, 2006.

[2] Y. P. Tsividis, "Accurate analysis of temperature effects in $\mathrm{I}_{\mathrm{C}}-\mathrm{V}_{\mathrm{BE}}$ characteristics with application to bandgap reference source," IEEE Journal of Solid-State Circuits, vol. 15, no. 6, pp. 1076-1084, 1980.

[3] W. Bludau, A. Onton, and W. Heinke, "Temperature dependence of the band gap of silicon," Journal of Applied Physics, vol. 45, no. 4, pp. 1846-1848, 1974.

[4] C. D. Thurmond, "The standard thermodynamic functions for the formation of electrons and holes in $\mathrm{Ge}, \mathrm{Si}, \mathrm{GaAs}$, and
GaP," Journal of the Electrochemical Society, vol. 122, no. 8, pp. 1133-1141, 1975.

[5] G. C. MacFarlane, J. P. McLean, J. E. Quarrington, and V. Roberts, "Fine structure in the absorption-edge spectrum of Si," Physical Review, vol. 111, no. 5, pp. 1245-1254, 1958.

[6] Y. P. Varshni, "Temperature dependence of the energy gap in semiconductors," Physica, vol. 34, no. 1, pp. 149-154, 1967.

[7] G. C. M. Meijer, Integrated circuits and components for bandgap references and temperature transducers, Ph.D. thesis, Delft University of Technology, The Netherlands, 1982.

[8] J. W. Slotboom and H. C. de Graaff, "Measurements of bandgap narrowing in Si bipolar transistors," Solid State Electronics, vol. 19, no. 10, pp. 857-862, 1976.

[9] R. Amador, A. Polanco, and A. Nagy, "Spread of $\eta$ and $\mathrm{V}_{\mathrm{G} 0}$ and its influence on the sensitivity of a bipolar IC Celsius sensor," Sensors and Actuators A, vol. 77, no. 1, pp. 9-13, 1999.

[10] A. Ohte and M. Yamagata, "A precision silicon transistor thermometer," IEEE Transactions on Instrumentation and Measurement, vol. 26, no. 4, pp. 335-341, 1977.

[11] A. van Staveren, C. J. M. Verhoeven, and A. H. M. van Roermund, "Influence of the reverse early effect on the performance of bandgap references," IEEE Transactions on Circuits and Systems I, vol. 43, no. 5, pp. 418-421, 1996.

[12] S. Mijalković, "Generalised early factor for compact modelling of bipolar transistors with non-uniform base," Electronics Letters, vol. 39, no. 24, pp. 1757-1758, 2003.

[13] M. Schroter, "HICUM, A scalable physics-based compact bipolar transistor model," October 2000, http://www.iee.et.tudresden.de/iee/eb/comp_mod.html.

[14] H. K. Gummel and H. C. Poon, "Integral charge control model of bipolar transistors," Bell System Technical Journal, vol. 49, no. 5, pp. 827-852, 1970.

[15] J. C. J. Paasschens and W. J. Kloosterman, "The Mextram Bipolar Transistor Model—Level 504,” Nat. Lab. Unclassified Report, NL-UR 2000/811, Philips NatLab.

[16] A. Nagy, A. Polanco, and M. Alvarez, "A new bipolar junction transistor base-emitter capacitance model," Sensor Letters, vol. 4, no. 3, pp. 305-311, 2006.

[17] W. Shockley, "The theory of p-n junctions in semiconductors and p-n junction transistors," Bell Systems Technical Journal, vol. 28, no. 3, pp. 435-489, 1949.

[18] S. Frégonèse, S. Lehmann, T. Zimmer et al., "A computationally efficient physics-based compact bipolar transistor model for circuit design-part II: parameter extraction and experimental results," IEEE Transactions on Electron Devices, vol. 53, no. 2, pp. 287-295, 2006.

[19] M. Schroter and H. Tran, "Charge-storage related parameter calculation for Si and SiGe bipolar transistors from device simulation," in Proceedings of the NSTI Nanotechnology Conference and Trade Show, pp. 735-740, May 2006.

[20] G. Wang and G. C. M. Meijer, "The temperature characteristics of bipolar transistors for CMOS temperature sensors," in Proceedings of the 13th European Conference on SolidState Transducers, pp. 553-556, The Hague, The Netherlands, September 1999.

[21] G. Wang and G. C. M. Meijer, "Temperature characteristics of bipolar transistors fabricated in CMOS technology," Sensors and Actuators A, vol. 87, no. 1-2, pp. 81-89, 2000.

[22] A. Nagy, A. Polanco, and M. Alvarez, "Contributions to improve design accuracy of bipolar ICs via physical effects," in Proceedings of the 20th Symposium on Integrated Circuits and Systems Design (SBCCI '07), Rio de Janeiro, Brazil, September 2007. 
[23] H. C. Poon and H. K. Gummel, "Modeling of emitter capacitance," Proceedings of the IEEE, vol. 57, no. 12, pp. 21812182, 1969.

[24] B. C. Bouma and A. C. Roelofs, "An experimental determination of the forward-biased emitter-base capacitance," Solid State Electronics, vol. 21, no. 6, pp. 833-836, 1978.

[25] I. E. Getreu, Modeling the Bipolar Transistor, Elsevier, New York, NY, USA, 1978.

[26] S. M. Sze, Physics of Semiconductor Devices, John Willey and Sons, Toronto, Canada, 1981.

[27] G. C. M. Meijer, "Thermal sensors based on transistors," Sensors and Actuators, vol. 10, no. 1-2, pp. 103-125, 1986. 

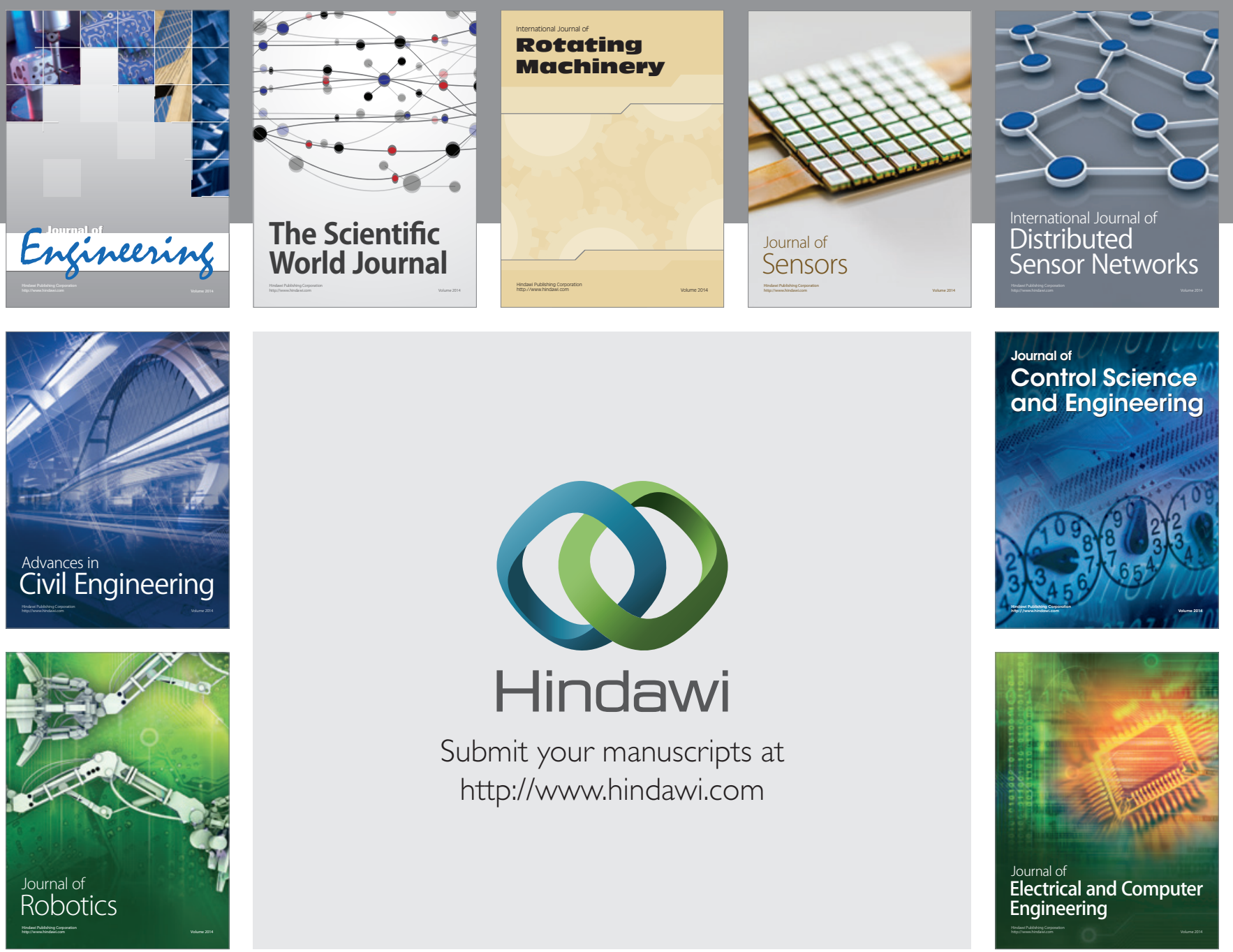

Submit your manuscripts at

http://www.hindawi.com
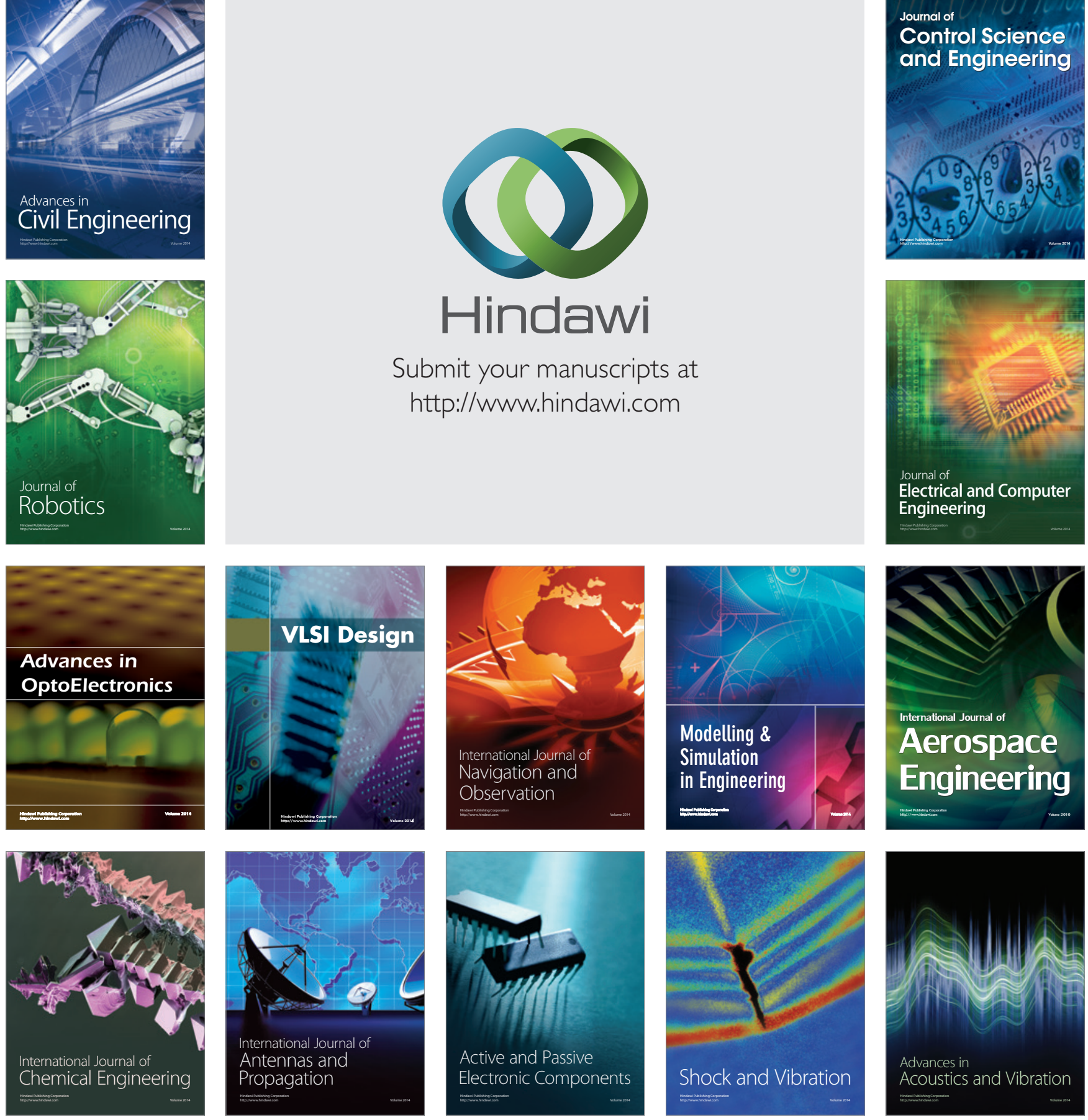